\title{
SOLUTIONS OF GENERALIZED FRACTIONAL KINETIC EQUATIONS VIA SUMUDU TRANSFORMS INVOLVING BESSEL-STRUVE KERNEL FUNCTION
}

\author{
KOTTAKKARAN S. NISAR ${ }^{1, *}$, GAUHAR RHAMAN ${ }^{2}$, FETHI BIN M. BELGACEM $^{3}$ \\ ABSTRACT. In this paper, we pursue and investigate the solutions for fractional kinetic \\ equations, involving Bessel-Struve function by means of their Sumudu transforms. In the \\ process, one Important special case is then revealed, and analyzed. The results obtained \\ in terms of Bessel-Struve function are rather general in nature and can easily construct \\ various known and new fractional kinetic equations.
}

\section{INTRODUCTION}

The Bessel-Struve kernel function $\mathfrak{S}_{\alpha}(\mu z), \mu, z \in \mathbb{C}[12]$ which is unique solution of the initial value problem $l_{\alpha} u(z)=\mu^{2} u(z)$ with the initial conditions $u(0)=1$ and $u^{\prime}(0)=\mu \Gamma(\alpha+1) / \sqrt{\pi} \Gamma(\alpha+3 / 2)$ is given by

$$
\mathfrak{S}_{\alpha}(\mu z)=j_{\alpha}(i \mu z)-i h_{\alpha}(i \mu z), \forall z \in \mathbb{C}
$$

where $j_{\alpha}$ and $h_{\alpha}$ are the normalized Bessel and Struve functions.

Moreover, the Bessel-Struve kernel function is a holomorphic function on $\mathbb{C} \times \mathbb{C}$ and it can be expanded in a power series:

$$
\mathfrak{S}_{\alpha}(\mu z)=\sum_{n=0}^{\infty} \frac{(\mu z)^{n} \Gamma(\alpha+1) \Gamma\left(\frac{n+1}{2}\right)}{\sqrt{\pi} n ! \Gamma\left(\frac{n}{2}+\alpha+1\right)} .
$$

For more details about Bessel-Struve kernel function interesting readers can see the references $[2,15,16]$.

The Sumudu transform introduced by Watugala (see $[27,28])$. For more details about Sumudu transform, see ( $[1,3-10])$.

The Sumudu transform over the set functions

$$
A=\left\{f(t)\left|\exists M, \tau_{1}, \tau_{2}>0,\right| f(t) \mid<M e^{|t| / \tau_{j}}, \text { if } t \in(-1)^{j} \times[0, \infty)\right\},
$$

Key words and phrases. Fractional Calculus; Bessel-Struve Function; Fractional Kinetic Equations; Sumudu Transforms.

${ }^{*}$ Corresponding author. 
is defined by

$$
G(u)=S[f(t) ; u]=\int_{0}^{\infty} f(u t) e^{-t} d t, u \in\left(-\tau_{1}, \tau_{2}\right) .
$$

The Sumudu transform of $\mathfrak{S}_{\alpha}(\mathfrak{x})$, using (1.2) and (1.3), is given by

$$
\begin{aligned}
S\left[\mathfrak{S}_{\alpha}(x)\right] & =\int_{0}^{\infty} e^{-t} \mathfrak{S}_{\alpha}(u t) d t \\
& =\int_{0}^{\infty} e^{-t} \sum_{n=0}^{\infty} \frac{(\mu)^{n} \Gamma(\alpha+1) \Gamma\left(\frac{n+1}{2}\right)}{\sqrt{\pi} n ! \Gamma\left(\frac{n}{2}+\alpha+1\right)}(u t)^{n} d t
\end{aligned}
$$

Interchanging the order of integration and summation gives,

$$
\begin{aligned}
S\left[\mathfrak{S}_{\alpha}(x)\right] & =\sum_{n=0}^{\infty} \frac{(\mu)^{n} u^{n} \Gamma(\alpha+1) \Gamma\left(\frac{n+1}{2}\right)}{\sqrt{\pi} n ! \Gamma\left(\frac{n}{2}+\alpha+1\right)} \int_{0}^{\infty} e^{-t} t^{n} d t \\
& =\sum_{n=0}^{\infty} \frac{(\mu)^{n} u^{n} \Gamma(\alpha+1) \Gamma\left(\frac{n+1}{2}\right) \Gamma(n+1)}{\sqrt{\pi} n ! \Gamma\left(\frac{n}{2}+\alpha+1\right)},
\end{aligned}
$$

Denoting the left hand side by $G(u)$, we have

$$
\begin{aligned}
G(u) & =S\left[\mathfrak{S}_{\alpha}(x) ; u\right] \\
& =\frac{\Gamma(\alpha+1)}{\sqrt{\pi}}{ }_{2} \Psi_{1}\left[\begin{array}{c}
\left(\frac{1}{2}, \frac{1}{2}\right),(1,1) \\
\left(\alpha+1, \frac{1}{2}\right)
\end{array} \mid \mu u\right] .
\end{aligned}
$$

The main aim of this work is to establish the generalized fractional kinetic equation involving $\mathfrak{S}_{\alpha}(x)$. Here, we use the Sumudu transform methodology to obtain the results.

\section{Generalized Fractional Kinetic Equations}

The fractional differential equation between rate of change of reaction was established in [13], the destruction rate and the production rate as follows

$$
\frac{d \mathfrak{N}}{d t}=-d\left(\mathfrak{N}_{t}\right)+p\left(\mathfrak{N}_{t}\right)
$$

where $\mathfrak{N}=\mathfrak{N}(t)$ the rate of reaction,$d=d(\mathfrak{N})$ the rate of destruction, $p=p(\mathfrak{N})$ the rate of production and $\mathfrak{N}_{t}$ denote the function defined by $\mathfrak{N}_{t}\left(t^{*}\right)=\mathfrak{N}\left(t-t^{*}\right), t^{*}>0$

The special case of (2.1), for spatial fluctuations or in homogeneities in $\mathfrak{N}(t)$ the quantity are neglected, that is the equation

$$
\frac{d \mathfrak{N}}{d t}=-c_{i} \mathfrak{N}_{i}(t)
$$

with t $\mathfrak{N}_{i}(t=0)=\mathfrak{N}_{0}$ is the number of density of species $i$ at time $t=0$ and $c_{i}>0$. If we reject the index $i$ and integrate the standard kinetic equation (2.2), we have

$$
\mathfrak{N}(t)-\mathfrak{N}_{0}=-c_{0} D_{t}^{-1} \mathfrak{N}(t)
$$


SOLUTIONS OF GENERALIZED FRACTIONAL KINETIC EQUATIONS VIA SUMUDU TRANSFORM.3 where ${ }_{0} D_{t}^{-1}$ is the special case of the Riemann-Liouville integral operator ${ }_{0} D_{t}^{-v}$ defined as

$$
{ }_{0} D_{t}^{-v} f(t)=\frac{1}{\Gamma(v)} \int_{0}^{t}(t-s)^{v-1} f(s) d s, t>0, \Re(v)>0
$$

The fractional generalization of the standard kinetic equation (2.3) given in [13] as:

$$
\mathfrak{N}(t)-\mathfrak{N}_{0}=-c_{0}^{v} D_{t}^{-1} \mathfrak{N}(t)
$$

and obtained the solution of (2.4) as follows

$$
\mathfrak{N}(t)=\mathfrak{N}_{0} \sum_{k=0}^{\infty} \frac{(-1)^{k}}{\Gamma(v k+1)}(c t)^{v k}
$$

Further, Saxena and Kalla [25] considered the following fractional kinetic equation:

$$
\mathfrak{N}(t)-\mathfrak{N}_{0} f(t)=-c^{v}\left({ }_{0} D_{t}^{-1} \mathfrak{N}\right)(t) \quad(\mathcal{R}(v)>0)
$$

where $\mathfrak{N}(t)$ denotes the number density of a given species at time $t, \mathfrak{N}_{0}=\mathfrak{N}(0)$ is the number density of that species at time $t=0, c$ is a constant and $f \in L(0, \infty)$. By applying the Laplace transform to (2.6), (see [25])

$$
L[\mathfrak{N}(t)](p)=\mathfrak{N}_{0} \frac{F(p)}{1+c^{v} p^{-v}}=\mathfrak{N}_{0}\left(\sum_{n=0}^{\infty}\left(-c^{v}\right)^{n} p^{-n v}\right) F(p) \quad\left(n \in \mathfrak{N}_{0},\left|\frac{c}{p}\right|<1\right)
$$

where the Laplace transform ( [26]) is defined by

$$
F(p)=L[f(t)]=\int_{0}^{\infty} e^{-p t} f(t) d t \quad \Re(p)>0
$$

The Mittag-Leffler functions $E_{\rho}(z)$ (see [14]) and $E_{\rho, \lambda}(x)[29]$ is defined respectively as

$$
\begin{gathered}
E_{\rho}(z)=\sum_{n=0}^{\infty} \frac{z^{n}}{\Gamma(\rho n+1)} \quad(z, \rho \in \mathbb{C} ;|z|<0, \Re(\rho)>0) . \\
E_{\rho, \lambda}(x)=\sum_{n=0}^{\infty} \frac{x^{n}}{\Gamma(\rho n+\lambda)} \quad(z, \rho, \lambda \in \mathbb{C} ; \Re(\rho)>0, \Re(\lambda)>0) .
\end{gathered}
$$

The details about fractional kinetic equations and solutions, one can refer to $[11,17-25,30]$ 
3. Solution of Generalized fRactional Kinetic EQuations involving (1.2)

The solution of the generalized fractional kinetic equations involving (1.2) is given in this section.

Theorem 1. If $d>0, v>0, \alpha, \mu, t \in \mathbb{C}$ and $\Re(\alpha)>-1$ then for the solution of the equation

$$
\mathfrak{N}(t)-\mathfrak{N}_{0} \mathfrak{S}_{\alpha}(\mu t)=-d^{v}{ }_{0} D_{t}^{-v} \mathfrak{N}(t)
$$

there holds the formula

$$
\mathfrak{N}(t)=\mathfrak{N}_{0} \sum_{n=0}^{\infty} \frac{\mu^{n} \Gamma(\alpha+1) \Gamma\left(\frac{n+1}{2}\right) t^{v n-1}}{\sqrt{\pi} \Gamma\left(\frac{n}{2}+\alpha+1\right)} E_{v, v n}\left(-d^{v} t^{v}\right)
$$

where $E_{v, n}($.$) is the generalized Mittag-Leffler function [14].$

Proof. Applying the Sumudu transform to the both sides of (3.1), gives

$$
\begin{gathered}
S[\mathfrak{N}(t) ; u]=\mathfrak{N}_{0} L\left[\mathfrak{S}_{\alpha}(\mu t) ; u\right]-d^{v} S\left[{ }_{0} D_{t}^{-v} \mathfrak{N}(t) ; u\right] \\
\mathfrak{N}^{*}(u)=\mathfrak{N}_{0}\left(\int_{0}^{\infty} e^{-t} \sum_{n=0}^{\infty} \frac{(\mu)^{n} \Gamma(\alpha+1) \Gamma\left(\frac{n+1}{2}\right)}{\sqrt{\pi} n ! \Gamma\left(\frac{n}{2}+\alpha+1\right)} t^{n} d t\right)-d^{v} u^{v} \mathfrak{N}^{*}(u) \\
\mathfrak{N}^{*}(u)+d^{v} u^{v} \mathfrak{N}^{*}(u)=\mathfrak{N}_{0} \sum_{n=0}^{\infty} \frac{(\mu)^{n} \Gamma(\alpha+1) \Gamma\left(\frac{n+1}{2}\right)}{\sqrt{\pi} n ! \Gamma\left(\frac{n}{2}+\alpha+1\right)} \int_{0}^{\infty} e^{-t} t^{n} d t \\
=\mathfrak{N}_{0} \sum_{n=0}^{\infty} \frac{(\mu)^{n} \Gamma(\alpha+1) \Gamma\left(\frac{n+1}{2}\right) \Gamma(n+1) u^{v n}}{\sqrt{\pi} n ! \Gamma\left(\frac{n}{2}+\alpha+1\right)} \\
\mathfrak{N}^{*}(u)=\mathfrak{N}_{0} \sum_{n=0}^{\infty} \frac{(\mu)^{n} \Gamma(\alpha+1) \Gamma\left(\frac{n+1}{2}\right) u^{v n}}{\sqrt{\pi} \Gamma\left(\frac{n}{2}+\alpha+1\right)} \sum_{r=0}^{\infty}\left[-(d u)^{v}\right]^{r}
\end{gathered}
$$

Taking Laplace inverse of (3.3), and by using $S^{-1}\left\{u^{v} ; t\right\}=\frac{t^{v-1}}{\Gamma(v)}, \Re(v)>0$, we get

$$
\begin{aligned}
S^{-1}(\mathfrak{N}(t))=\mathfrak{N}_{0} \sum_{n=0}^{\infty} \frac{(\mu)^{n} \Gamma(\alpha+1) \Gamma\left(\frac{n+1}{2}\right)}{\sqrt{\pi} \Gamma\left(\frac{n}{2}+\alpha+1\right)} S^{-1}\left\{\sum_{r=0}^{\infty} d^{v r} u^{(v n+v r)}\right\} \\
\mathfrak{N}(t)=\mathfrak{N}_{0} \sum_{n=0}^{\infty} \frac{(\mu)^{n} \Gamma(\alpha+1) \Gamma\left(\frac{n+1}{2}\right)}{\sqrt{\pi} \Gamma\left(\frac{n}{2}+\alpha+1\right)}\left\{\sum_{r=0}^{\infty}(-1)^{r} d^{v r} \frac{t^{(v n+v r)-1}}{\Gamma(v n+v r)}\right\} \\
=\mathfrak{N}_{0} \sum_{n=0}^{\infty} \frac{(\mu)^{n} \Gamma(\alpha+1) \Gamma\left(\frac{n+1}{2}\right)}{\sqrt{\pi} \Gamma\left(\frac{n}{2}+\alpha+1\right)} t^{v n-1}\left\{\sum_{r=0}^{\infty}(-1)^{r} d^{v r} \frac{t^{v r}}{\Gamma(v n+v r)}\right\}
\end{aligned}
$$

In view of equation (2.9), we obtain the desired result. 
SOLUTIONS OF GENERALIZED FRACTIONAL KINETIC EQUATIONS VIA SUMUDU TRANSFORM.5

Theorem 2. If $d>0, v>0, \alpha, \mu, t \in \mathbb{C}$ and $\mathcal{R}(\alpha)>-1$, then for the solution of the equation

$$
\mathfrak{N}(t)-\mathfrak{N}_{0} \mathfrak{S}_{\alpha}\left(\mu d^{v} t^{v}\right)=-d^{v}{ }_{0} D_{t}^{-v} \mathfrak{N}(t)
$$

there holds the formula

$$
\mathfrak{N}(t)=\mathfrak{N}_{0} \sum_{n=0}^{\infty} \frac{\Gamma(\alpha+n) \Gamma\left(\frac{n+1}{2}\right) \Gamma(v n+1)\left(\mu d^{v}\right)^{n} t^{v n-1}}{\sqrt{\pi} n ! \Gamma\left(\frac{n}{2}+\alpha+1\right)} E_{v, v n}\left(-d^{v} t^{v}\right)
$$

Proof. This theorem can prove parallel as Theorem 1. So the details are omitted.

Theorem 3. If $d>0, v>0, \alpha, \mu, t \in \mathbb{C}, \mathfrak{a} \neq d$ and $\Re(\alpha)>-1$, then for the solution of the equation

$$
\mathfrak{N}(t)-\mathfrak{N}_{0} \mathfrak{S}_{\alpha}\left(\mu d^{v} t^{v}\right)=-\mathfrak{a}^{v}{ }_{0} D_{t}^{-v} \mathfrak{N}(t)
$$

there holds the formula

$$
\mathfrak{N}(t)=\mathfrak{N}_{0} \sum_{n=0}^{\infty} \frac{\Gamma(\alpha+n) \Gamma\left(\frac{n+1}{2}\right) \Gamma(v n+1)\left(\mu d^{v}\right)^{n} t^{v n-1}}{\sqrt{\pi} n ! \Gamma\left(\frac{n}{2}+\alpha+1\right)} E_{v, v n}\left(-\mathfrak{a}^{v} t^{v}\right)
$$

Proof. Theorem 3 can easily derive from Theorem 2, so the details are omitted.

\section{Special case:}

If we set $\alpha=-\frac{1}{2}$ in (1.2), we have

$$
\begin{aligned}
\mathfrak{S}_{-\frac{1}{2}}(\mu z) & =\sum_{n=0}^{\infty} \frac{(\mu z)^{n} \Gamma\left(-\frac{1}{2}+1\right) \Gamma\left(\frac{n+1}{2}\right)}{\sqrt{\pi} n ! \Gamma\left(\frac{n}{2}-\frac{1}{2}+1\right)} \\
, & =\sum_{n=0}^{\infty} \frac{(\mu z)^{n} \Gamma\left(\frac{1}{2}\right) \Gamma\left(\frac{n+1}{2}\right)}{\sqrt{\pi} n ! \Gamma\left(\frac{n}{2}+\frac{1}{2}\right)} \\
& =\sum_{n=0}^{\infty} \frac{(\mu z)^{n}}{n !}
\end{aligned}
$$

which implies that

$$
\mathfrak{S}_{-\frac{1}{2}}(\mu z)=e^{\mu z}
$$

In view of (3.8) and Theorem 1, 2 and 3, we have following corollaries respectively

Corollary 3.1. If $d>0, v>0, \alpha, \mu, t \in \mathbb{C}$ then for the solution of the equation

$$
\mathfrak{N}(t)-\mathfrak{N}_{0} \mathfrak{S}_{-\frac{1}{2}}(\mu t)=-d^{v}{ }_{0} D_{t}^{-v} \mathfrak{N}(t)
$$

there holds the formula

$$
\mathfrak{N}(t)=\mathfrak{N}_{0} \sum_{n=0}^{\infty} \frac{\mu^{n} t^{v n-1} \Gamma(v n+1)}{n !} E_{v, v n}\left(-d^{v} t^{v}\right)
$$


Corollary 3.2. If $d>0, v>0, \alpha, \mu, t \in \mathbb{C}$ then for the solution of the equation

$$
\mathfrak{N}(t)-\mathfrak{N}_{0} \mathfrak{S}_{-\frac{1}{2}}\left(\mu d^{v} t^{v}\right)=-d^{v}{ }_{0} D_{t}^{-v} \mathfrak{N}(t)
$$

there holds the formula

$$
\mathfrak{N}(t)=\mathfrak{N}_{0} \sum_{n=0}^{\infty} \frac{\left(\mu d^{v}\right)^{n} t^{v n-1}}{n !} E_{v, v n}\left(-d^{v} t^{v}\right)
$$

Corollary 3.3. If $d>0, v>0, \mu, t \in \mathbb{C}$ and $\mathfrak{a} \neq d$ then for the solution of the equation

$$
\mathfrak{N}(t)-\mathfrak{N}_{0} \mathfrak{S}_{-\frac{1}{2}}\left(\mu d^{v} t^{v}\right)=-\mathfrak{a}^{v}{ }_{0} D_{t}^{-v} \mathfrak{N}(t)
$$

there holds the formula

$$
\mathfrak{N}(t)=\mathfrak{N}_{0} \sum_{n=0}^{\infty} \frac{\left(\mu d^{v}\right)^{n} t^{v n-1}}{n !} E_{v, v n}\left(-\mathfrak{a}^{v} t^{v}\right)
$$

\section{Conclusion}

Solutions of generalized fractional kinetic equation in terms of the Bessel-Struve kernel function is given in this study. The results obtained here are in compact forms appropriate for numerical computation and are rather general in nature and can easily construct various known and new fractional kinetic equations.

\section{REFERENCES}

[1] M.A. Asiru, Sumudu transform and the solution of integral equation of convolution type. International Journal of Mathematical Education in Science and Technology. 32, 906-910(2001)

[2] A. Baricz, S.R. Mondal, A. Swaminathan, Monotonicity properties of the Bessel-Struve kernel. Bulletin of the Korean Mathematical Society,(In Press), (2016)

[3] F.B.M. Belgacem, A.A. Karaballi, S.L. Kalla, Analytical Investigations of the Sumudu Transform and Applications to Integral Production Equations. Journal of Mathematical Problems in Engineering. $3,103-118(2003)$

[4] F.B.M. Belgacem, A.A. Karaballi, Sumudu transform fundamental properties investigations and applications. International J. Appl. Math. Stoch. Anal. (2005) 1-23.

[5] F.B.M. Belgacem, A.A. Karaballi, Sumudu Transform Fundamental Properties Investigations and Applications. Journal of Applied Mathematics and Stochastic Analysis. 2006,1-23(2006)

[6] F.B.M. Belgacem, Introducing and Analyzing Deeper Sumudu Properties. Nonlinear Studies. 13, 23-42(2006)

[7] F.B.M. Belgacem, Applications with the Sumudu Transform to Bessel Functions and Equations. Applied Math. Sciences. 4, 3665-3686(2010)

[8] F.B.M. Belgacem, Sumudu applications to Maxwell's equations. PIERS Online, 5, 355-360(2009)

[9] F.B.M. Belgacem, E.H. Al-Shemas, R. Silambarasan, Sumudu computation of the transient magnetic field in a lossy medium. Applied Mathematics \& Information Sciences. 6, 1-9(2016) 


\section{SOLUTIONS OF GENERALIZED FRACTIONAL KINETIC EQUATIONS VIA SUMUDU TRANSFORM.7}

[10] F.B.M. Belgacem, R. Silambarasan, A distinctive Sumudu treatment of trigonometric functions. Journal of Computational and Applied Mathematics. 312, 74-81(2017)

[11] V.B.L. Chaurasia and S.C. Pandey, On the new computable solution of the generalized fractional kinetic equations involving the generalized function for the fractional calculus and related functions, Astrophys. Space Sci., 317 (2008), 213-219.

[12] A. Gasmi and M. Sifi, The Bessel-Struve intertwinning operator on C and mean-periodic functions, IJMMS 2004:59, 3171-3185

[13] H.J. Haubold and A.M. Mathai, The fractional kinetic equation and thermonuclear functions, Astrophys. Space Sci., 327 (2000), 53-63.

[14] Mittag-Leffler, G.M.: Sur la representation analytiqie d'une fonction monogene cinquieme note. Acta Math. 29, 101-181(1905)

[15] S.R. Mondal, On the geometric properties of the Bessel-Struve kernel function, arXiv:1601.08102 [math.CV], (2016)

[16] K. S. Nisar, S. R. Mondal and P. Agarwal, Composition Formulas of Bessel-Struve Kernel Function, Mathematical Problems in Engineering, 2016 (2016), doi.org/10.1155/2016/9560346

[17] K.S. Nisar, S.D. Purohit, S.R. Mondal, Generalized fractional kinetic equations involving generalized Struve function of the first kind. Journal of King Saud University Science, 28, 167-171(2016)

[18] K.S. Nisar, D. Baleanu, M. Alqurasi, Fractional Calculus and application of generalized Struve functions. SpringerPlus, 5, 910(2016)

[19] K.S. Nisar, F.B.M. Belgacem, Dynamic k-Struve Sumudu solutions for fractional kinetic equations. Advances in Difference Equations 2017:340 (2017).

[20] K. S. Nisar, F.Qi, On solutions of fractional kinetic equations involving the generalized k-Bessel function, Note di Matematica 37, 1-10 (2017).

[21] A. Saichev, M. Zaslavsky, Fractional kinetic equations: solutions and applications, Chaos, 7(1997), 753-764.

[22] R.K. Saxena, A.M. Mathai, H.J. Haubold, On Fractional kinetic equations, Astrophys. Space Sci., 282 (2002), 281-287.

[23] R.K. Saxena, A.M. Mathai, H.J. Haubold, On generalized fractional kinetic equations, Physica A 344 (2004), 657-664.

[24] R.K. Saxena, A.M. Mathai, H.J. Haubold, Solution of generalized fractional reaction-diffusion equations, Astrophys. Space Sci., 305 (2006), 305-313.

[25] R.K. Saxena, S.L. Kalla, On the solutions of certain fractional kinetic equations, Appl. Math. Comput., 199 (2008), 504-511.

[26] M.R. Spiegel, Theory and Problems of Laplace Transforms, Schaums Outline Series, McGraw-Hill, New York, 1965.

[27] G.K. Watugala, Sumudu transform:a new integral transform to solve differential equations and control engineering problems. Int.J.Math. Edu.Sci.Tech. 24, 35-43(1993)

[28] G.K. Watugala, The Sumudu transform for functions of two variables, Math. Eng.Ind. 8, 293$302(2002)$

[29] Wiman, A.: Uber den fundamental satz in der theorie der funktionen $E_{\alpha}(z)$. Acta Math. 29, 191201(1905)

[30] G.M. Zaslavsky, Fractional kinetic equation for Hamiltonian chaos, Physica D, 76(1994), 110-122. 
8 K.S. NISAR, G. RAHMAN, F.B.M. BELGACEM

K. S. Nisar : 1 Department of Mathematics, College of Arts and Science-Wadi AlDaWaser, 11991, Prince Sattam bin Abdulaziz University, Alkharj, Saudi Arabia, orcid.org/00000001-5769-4320

E-mail address: ksnisar1@gmail.com, n.sooppy@psau.edu.sa

G.Rahman: ${ }^{2}$ Department of Mathematics, International Islamic University, Pakistan E-mail address: gauhar55uom@gmail.com

F.B.M. Belgacem: ${ }^{3}$ Department of Mathematics, Faculty of Basic Education, PAAET, Al-ArDhiya, KuWAit.

E-mail address: fbmbelgacem@gmail.com 